International Mathematical Forum, Vol. 9, 2014, no. 29, 1369 - 1375

HIKARI Ltd, www.m-hikari.com

http://dx.doi.org/10.12988/imf.2014.47131

\title{
Diameter of the Zero Divisor Graph of Semiring of Matrices over Boolean Semiring
}

\author{
L. John
}

Department of Mathematics, University of Kerala, Trivandrum, India

\section{Harita Vijay}

Department of Mathematics, University of Kerala, Trivandrum, India

Copyright (c) 2014 L. John and Harita Vijay. This is an open access article distributed under the Creative Commons Attribution License, which permits unrestricted use, distribution, and reproduction in any medium, provided the original work is properly cited.

\begin{abstract}
Let $S$ be a semiring and let $Z(S)^{*}$ be its set of nonzero zero divisors. We denote the zero divisor graph of $S$ by $\Gamma(S)$ whose vertex set is $Z(S)^{*}$ and there is an edge between the vertices $x$ and $y(x \neq y)$ in $\Gamma(S)$ if and only if either $x y=0$ or $y x=0$. In this paper we study the zero divisor graph of the semiring of matrices $M_{n}(\mathfrak{B}),(n>1)$ over the Boolean semiring $\mathfrak{B}$. We investigate the properties of the right zero divisors and the left zero divisors of $M_{n}(\mathfrak{B})$ and then use these results to prove that the diameter of $\Gamma\left(M_{n}(\mathfrak{B})\right)$ is 3 .
\end{abstract}

Mathematics Subject Classification: 5C25

Keywords: Zero divisor graph, semiring of matrices, Boolean semiring, zero divisor, diameter of a graph

\section{Introduction}

The concept of a zero divisor graph was first introduced by Beck [5] in the study of commutative rings and later redefined by Anderson and Livingston [3] . Redmond [11] extended this concept to the non commutative case. Dolzan and Oblak [8] further extended the idea to semirings. For further results on zero divisor graphs see [1], [2], [4], [6], [7], [10]. 
A semiring is a nonempty set $S$ on which the operations of + and $\times$ have been defined such that the following conditions are satisfied (see [9]).

1. $(S,+)$ is a commutative monoid with identity element 0 .

2. $(S,$.$) is a monoid with identity element 1$.

3. Multiplication distributes over addition from either side.

4. $0 s=0=s 0$, for all $s \in S$.

Here $1 \neq 0$, to avoid the trivial case. Again 0 is the only absorbing zero, for if $z \in S$ satisfy $z s=z=s z, \forall s \in S$ then $0=0 z=z$. For any semiring $S$, we denote the set of zero divisors by $Z(S)$, that is, $Z(S)=\{x \in S$ : there exists $0 \neq y \in S$ such that $x y=0$ or $y x=0\}$. Then $Z_{R}(S)$ denotes the set of right zero divisors, that is, $Z_{R}(S)=\{x \in S$ : there exists $0 \neq y \in S$ such that $y x=0\}$ and $Z_{L}(S)$ denotes the set of left zero divisors, that is, $Z_{L}(S)=\{x \in S$ : there exists $0 \neq y \in S$ such that $x y=0\}$. We associate a zero divisor graph to the semiring $S$, denoted by $\Gamma(S)$ whose vertex set is the set of all non zero, zero divisors of $S$. That is, the vertex set $V(\Gamma(S))$ of $\Gamma(S)$ is the set of elements in $Z(S)^{*}=Z(S)-\{0\}$. An unordered pair of vertices $x, y \in V(\Gamma(S)), x \neq y$ is an edge in $\Gamma(S)$ if and only if either $x y=0$ or $y x=0$. That is, we consider the graph $\Gamma(S)$ whose vertices are the elements of $Z(S)^{*}$ and whose edges are those pairs of distinct non zero zero divisors $x, y$ such that either $x y=0$ or $y x=0$.

\section{Diameter of $\Gamma\left(M_{n}(\mathfrak{B})\right)$}

We recall that a graph is connected if there exists a path connecting any two distinct vertices. The distance between two distinct vertices $x$ and $y$, denoted by $d(x, y)$ is the length of the shortest path connecting them. The diameter of a graph $\Gamma$, denoted by $\operatorname{diam}(\Gamma)$ is equal to $\sup \{d(x, y): x, y$ distinct vertices of $\Gamma$. Dolzan and Oblak [8] proved the following theorem:

Theorem 2.1 For a semiring $S$, the zero divisor graph $\Gamma(S)$ is always connected and its diameter, $\operatorname{diam}(\Gamma(S)) \leq 3$.

We denote the Boolean semiring by $(\mathfrak{B},+,$.$) where \mathfrak{B}=\{0,1\}$ and the operations of + and are defined as follows: $0+0=0,0+1=1,1+0=$ $1,1+1=1,0.0=0,0.1=0,1.0=0,1.1=1$. The semiring of all $n \times n$ matrices over $\mathfrak{B}$ is denoted by $M_{n}(\mathfrak{B})$, where $n>1$. The matrix with the only non zero entry 1 in the $i$ th row and $j$ th column will be denoted by $E_{i, j}$.

Proposition 2.1 If $A, B \in M_{n}(\mathfrak{B})$ and $A$ is a non-zero matrix and $B$ is a matrix with all rows as non-zero rows then their product $A B$ will be a non-zero matrix. 
Proof. Without loss of generality let us assume that $A=E_{p, q}$. Let $B$ be the matrix with all rows as non-zero rows. ie., $B=\left[b_{j k}\right]$ where for each $j=1,2, \ldots, n, b_{j k}=1$, for atleast one $k=1,2, \ldots, n$. Then the $p$ th row in the product $A B$ is $\left[\begin{array}{llll}b_{q 1} & b_{q 2} & \ldots & b_{q n}\end{array}\right]$. Since $b_{q k}=1$ for atleast one $k=1,2, \ldots, n$ the above row is a non-zero row. This shows that $A B$ is a non-zero matrix.

Theorem 2.2 Every right zero divisor should have atleast one zero row.

Proof. Let $B$ be a right zero divisor. Then there exists a non zero matrix $A$ such that $A B=0$. If possible, let $B$ be such that $B$ has all the rows as non-zero rows. Then by proposition $2.1, A B \neq 0$, contradicting the fact that $A B=0$. Hence $B$ has atleast one zero row.

Theorem 2.3 Any matrix of $M_{n}(\mathfrak{B})$ having atleast one zero row is a right zero divisor.

Proof. Consider a matrix $B \in M_{n}(\mathfrak{B})$ having only one zero row, say the $i$ th row. Then there exists a matrix $E_{i, i}$ such that $E_{i, i} B=0$. Hence $B$ is a right zero divisor. Similarly we can prove that matrices of $M_{n}(\mathfrak{B})$ having 2 zero rows, 3 zero rows,..., $(n-1)$ zero rows are all right zero divisors. Thus any matrix of $M_{n}(\mathfrak{B})$ having atleast one zero row is a right zero divisor.

Proposition 2.2 If $A, B \in M_{n}(\mathfrak{B})$ and $A$ is a matrix with all the columns as non-zero columns and $B$ is a non-zero matrix then their product $A B$ will be a non-zero matrix.

Proof. Without loss of generality let us assume that the non-zero matrix $B=E_{p q}$. Let $A$ be the matrix with all columns as non-zero columns. ie., $A=\left[a_{i j}\right]$, where for each $j=1,2, \ldots, n, a_{i j}=1$ for atleast one $i=1,2, \ldots, n$. Then the $q$ th column in the product $A B$ is $\left[\begin{array}{llll}a_{1 p} & a_{2 p} & \ldots & a_{n p}\end{array}\right]^{T}$. Since $a_{i p}=1$ for atleast one $i=1,2, \ldots, n$ the above column is a non-zero column. This shows that $A B$ is a non-zero matrix.

Theorem 2.4 Every left zero divisor should have atleast one zero column.

Proof. Let $A$ be a left zero divisor. Then there exists a non-zero matrix $B \in M_{n}(\mathfrak{B})$ such that $A B=0$. If possible let $A$ be such that, $A$ has all columns as non-zero columns. Then by proposition $2.2, A B \neq 0$, contradicting the fact that $A B=0$. Hence $A$ has atleast one zero column.

Theorem 2.5 Any matrix of $M_{n}(\mathfrak{B})$ having atleast one zero column is a left zero divisor.

Proof. Consider a matrix $A \in M_{n}(\mathfrak{B})$ having only one zero column, say the $i$ th column. Then there exists a matrix $E_{i, i}$ such that $A E_{i, i}=0$. Hence $A$ is a left zero divisor. Similarly we can prove that matrices of $M_{n}(\mathfrak{B})$ having 2 zero columns, 3 zero columns, $\ldots,(n-1)$ zero columns are all left zero divisors. Thus any matrix of $M_{n}(\mathfrak{B})$ having atleast one zero column is a left zero divisor. 
Corollary 2.6 $E_{i, j}$ is both right zero divisor and left zero divisor.

Proof. Follows from theorem 2.3 and theorem 2.5

Lemma 2.7 If $x$ is a left zero divisor with pth column as the only zero column then $x E_{p, j}=0$, for $j=1,2, \ldots, n$.

Proof. Let $x=\left[x_{i j}\right]$ for $i=1,2, \ldots, n$ and $j=1,2, \ldots, n$. Since the $p$ th column of $x$ is a zero column, we have $x_{i p}=0$, for $i=1,2, \ldots, n$. Let $E_{p, j}=\left[e_{i j}\right], i=1,2, \ldots, n, j=1,2, \ldots, n$, where $e_{p j}=1$ and $e_{i j}=0$ otherwise. Then the $(i, j)$ th element of $x E_{p, j}=\sum_{k=1}^{n} x_{i k} e_{k j}=x_{i p}=0$. Therefore $x E_{p, j}=0$, for $j=1,2, \ldots, n$.

Lemma 2.8 If $y$ is a right zero divisor with qth row as the only zero row then $E_{i, q} y=0$, for $i=1,2, \ldots, n$.

Proof. Let $y=\left[y_{i j}\right]$ for $i=1,2, \ldots, n$ and $j=1,2, \ldots, n$. Since the $q$ th row of $y$ is a zero row, we have $y_{q j}=0$, for $j=1,2, \ldots, n$. Let $E_{i, q}=\left[e_{i j}\right], i=$ $1,2, \ldots, n, j=1,2, \ldots, n$, where $e_{i q}=1$ and $e_{i j}=0$ otherwise. Then the $(i, j)$ th element of $E_{i, q} y=\sum_{k=1}^{n} e_{i k} y_{k j}=y_{q j}=0$. Therefore $E_{i, q} y=0$, for $i=1,2, \ldots, n$.

Let $R$ denote the set of all right zero divisors of $M_{n}(\mathfrak{B})$ and $L$ the set of all left zero divisors of $M_{n}(\mathfrak{B})$. Let $R^{\prime}=R-(R \cap L)$ and $L^{\prime}=L-(R \cap L)$. Then for $d(x, y)$ where $x, y \in V\left(\Gamma\left(M_{n}(\mathfrak{B})\right)\right)$ there arise three exhaustive cases. Case 1: $x \in L, y \in R$.

Case 2: $x \in R^{\prime}, y \in R^{\prime}$.

Case 3: $x \in L^{\prime}, y \in L^{\prime}$.

Theorem 2.9 If $x \in L, y \in R$, then $d(x, y) \leq 2$.

Proof. Case 1: For $x \in L$, if $y$ is that right zero divisor so that $x y=0$ then $d(x, y)=1$.

Case 2: Suppose that $x y \neq 0$. Then $d(x, y) \neq 1$. By theorem 2.4, since a left zero divisor should have atleast one zero column, without loss of generality let us assume that the $p$ th column of $x$ is the only zero column. Then by lemma $2.7, x E_{p, j}=0$ for $j=1,2, \ldots, n$. Therefore, $d\left(x, E_{p, j}\right)=1$, for $j=1,2, \ldots, n$. By theorem 2.2, a right zero divisor should have atleast one zero row. If the $q$ th row of $y$ is the only zero row then by lemma $2.8, E_{i, q} y=0$, for $i=1,2, \ldots, n$. Therefore, $d\left(E_{i, q} y\right)=1$, for $i=1,2, \ldots, n$. In particular $E_{p, q} y=0$. Hence $x E_{p, q} y=0$. Thus $d(x, y)=2$. 
Lemma 2.10 If $x$ is a right zero divisor with the pth row as the only zero row then any left zero divisor of $x$ should have non-zero elements only in the pth column.

Proof. Let $x=\left[x_{i j}\right]$, for $i=1,2, \ldots, n, j=1,2, \ldots, n$ where $x_{p j}=0$ for $j=$ $1,2, \ldots, n$. Consider $z \in V\left(\Gamma\left(M_{n}(\mathfrak{B})\right)\right)$ with $z=\left[z_{i j}\right]$, for $i=1,2, \ldots, n, j=$ $1,2, \ldots, n$. Now the $(i, j)$ th element in the product $z x$ is $\sum_{k=1}^{n} z_{i k} x_{k j}$. Suppose $z$ is a left zero divisor of $x$ with the $t$ th column as a zero column. Then we know that $z x=0$. That is, each $(i, j)$ th element of $z x$ is zero. That is, the product $z_{i k} x_{k j}=0$ for each $i, j, k=1,2, \ldots, n$. If possible, let $z$ contain non-zero elements in the $m$ th column, where $m \neq p$. Since by assumption $z$ contains non-zero elements in the $m$ th column, without loss of generality we assume that $z_{l m}$ is a non-zero element in $z$. Then in the product $z x$ there will be sums involving terms $z_{l m} x_{m j}, j=1,2, \ldots, n$. Since the $p$ th row is the only zero row in $x, x_{m j} \neq 0$, for atleast one $j=1,2, \ldots, n$. Hence let us suppose that $x_{m q} \neq 0$. Then $z_{l m} x_{m q} \neq 0$. Therefore, $z x \neq 0$. This is a contradiction to the hypothesis that $z$ is a left zero divisor of $x$. Hence $z$ is a left zero divisor of $x$ with non-zero elements only in the $p$ th column.

Theorem 2.11 Let $x, y \in R^{\prime}$

1.If the positions of zero rows of $x$ and $y$ are non-overlapping then $d(x, y)=3$. 2.If the positions of zero rows of $x$ and $y$ are overlapping then $d(x, y)=2$.

Proof. Let $x, y \in R^{\prime}$. Then $d(x, y) \neq 1$.

1. Suppose the positions of zero rows of $x$ and $y$ are non-overlapping. Without loss of generality assume that the $p$ th row of $x$ is the zero row and the $q$ th row of $y$ is the zero row. Then, by lemma 2.10, any left zero divisor of $x$ has nonzero elements only in the $p$ th column and any left zero divisor of $y$ has non-zero elements only in the $q$ th column. ie., $x$ and $y$ do not have any common left zero divisors. Thus $d(x, y) \neq 2$. Hence by Theorem $2.1, d(x, y)=3$.

2. Suppose the positions of zero rows of $x$ and $y$ are overlapping. Without loss of generality assume that the $p$ th row of $x$ and the $p$ th row of $y$ are the zero rows. Then by lemma 2.10, they have left zero divisors with non-zero elements only in the $p$ th column. That is, they have common left zero divisors. Thus $d(x, y)=2$.

Lemma 2.12 If $x$ is a left zero divisor with the qth column as the only zero column then any right zero divisor of $x$ should have non-zero elements only in the qth row.

Let $x=\left[x_{i j}\right]$, for $i=1,2, \ldots, n, j=1,2, \ldots, n$ where $x_{i q}=0$ for $i=$ $1,2, \ldots, n$. Consider $z \in V\left(\Gamma\left(M_{n}(\mathfrak{B})\right)\right)$ with $z=\left[z_{i j}\right]$, for $i=1,2, \ldots, n, j=$ 
$1,2, \ldots, n$. Now the $(i, j)$ th element in the product $x z$ is $\sum_{k=1}^{n} x_{i k} z_{k j}$. Suppose $z$ is a right zero divisor of $x$ with the $t$ th row as a zero row. Then we know that $x z=0$. That is, each $(i, j)$ th element of $x z$ is zero. That is, the product $x_{i k} z_{k j}=0$ for each $i, j, k=1,2, \ldots, n$. If possible let $z$ contain non-zero elements in the $m$ th row, where $m \neq q$. Since by assumption $z$ contains nonzero elements in the $m$ th row, without loss of generality we assume that $z_{m l}$ is a non-zero element in $z$. Then in the product $x z$ there will be sums involving terms $x_{i m} z_{m l}, i=1,2, \ldots, n$. Since the $q$ th column is the only zero column in $x, x_{i m} \neq 0$, for atleast one $i=1,2, \ldots, n$. Hence let us suppose that $x_{q m} \neq 0$. Then $x_{q m} z_{m l} \neq 0$. Therefore, $x z \neq 0$. This is a contradiction to the hypothesis that $z$ is a right zero divisor of $x$.Hence $z$ is a right zero divisor of $x$ with non-zero elements only in the $q$ th row.

Theorem 2.13 Let $x, y \in L^{\prime}$.

1.If the positions of zero columns of $x$ and $y$ are non-overlapping then $d(x, y)=3$.

2.If the positions of zero columns of $x$ and $y$ are overlapping then $d(x, y)=2$.

Proof. Let $x, y \in L^{\prime}$. Then $d(x, y) \neq 1$.

1. Suppose the positions of zero columns of $x$ and $y$ are non-overlapping. Without loss of generality assume that the $p$ th column of $x$ is the zero column and the $q$ th column of $y$ is the zero column. Then by lemma 2.12 any right zero divisor of $x$ has non-zero elements only in the $p$ th row and any right zero divisor of $y$ has non-zero elements only in the $q$ th row. ie., $x$ and $y$ do not have any common right zero divisors. Thus $d(x, y) \neq 2$. Hence by Theorem $2.1, d(x, y)=3$.

2. Suppose the positions of zero columns of $x$ and $y$ are overlapping. Without loss of generality assume that the $p$ th column of $x$ and the $p$ th column of $y$ are the zero columns. Then by lemma 2.12 , they have right zero divisors with non-zero elements only in the $p$ th row. That is, they have common right zero divisors. Thus $d(x, y)=2$.

Theorem 2.14 The diameter of $\Gamma\left(M_{n}(\mathfrak{B})\right)$ is 3 .

Proof. Follows from theorem 2.9, theorem 2.11 and theorem 2.13.

\section{References}

[1] D.F. Anderson, On the diameter and girth of a zero-divisor graph. II, Houston J. Math., 34 (2008), no. 2, 361-371.

[2] D.F. Anderson and A. Badawi, On the zero-divisor graph of a ring, Comm. Algebra, 36 (2008), no. 8, 3073-3092. 
[3] D.F. Anderson and P.S. Livingston, The zero-divisor graph of a commutative ring, J. Algebra, 217 (1999), 434-447.

[4] D.F. Anderson and S.B. Mulay, On the diameter and girth of a zero-divisor graph, J. Pure Appl. Alg., 210 (2007), 543-550.

[5] I. Beck, Coloring of commutative rings, J. Algebra, 116 (1988), 208-226.

[6] I. Bozic and Z. Petrovic, Zero-divisor graphs of matrices over commutative rings, Comm. Algebra, 37 (2009), no. 4, 1186-1192.

[7] F.R. DeMeyer, T. McKenzie and K. Schneider, The zero-divisor graph of a commutative semi-group, Semigroup Forum, 65 (2002), 206-214.

[8] D. Dolzan and P. Oblak, The zero-divisor graphs of rings and semi-rings, Int. J. Algebra and Computation, 22 (2012), no. 4, 1250033, 20 str.

[9] Jonathan S. Golan, Semirings and their applications , Kluwer Academic Publishers, Dordrecht, 1999.

[10] T.G. Lucas, The diameter of a zero divisor graph, J. Algebra, 301 (2006), 174-193.

[11] S.P. Redmond, The zero divisor graph of a non-commutative ring, Int. J. Commut. Rings, 1 (2002), no. 4, (1999), 203-211.

Received: July 11, 2014 